\title{
Determination of hygrothermal parameters of experimental and commercial bio-based insulation materials
}

\author{
M. Palumbo ${ }^{\text {a* }}$, A.M. Lacasta ${ }^{\mathrm{a}}$, N. Holcroft ${ }^{\mathrm{b}}$, A. Shea ${ }^{\mathrm{b}}$ and P. Walker ${ }^{\mathrm{b}}$ \\ ${ }^{a}$ Escola Politècnica Superior d'Edificació de Barcelona, Departament de Física Aplicada, Universitat \\ Politècnica de Catalunya, Barcelona, Spain. \\ *Corresponding author: UPC-EPSEB, Av Dr. Marañon 44-50 08028 Barcelona \\ Tel: $+34 / 669580593$ \\ E-mail: mariana.palumbo@upc.edu \\ ${ }^{b}$ BRE CICM, Department of Architecture and Civil Engineering, University of Bath, Bath, UK
}

\begin{abstract}
The development and application of bio-based insulation materials can contribute to the minimization of the environmental impacts of buildings through reduction of embodied and in-use energy demand, in addition to many other major impacts such as resource depletion and waste generation. The hygrothermal performance of natural building materials has direct and indirect impacts on moderating indoor environmental conditions and can contribute to energy savings provided that such aspects are taken into account during the design and construction phases. This requires in-depth knowledge of the thermal and hygroscopic properties of the materials and their dependence on the moisture content. In this paper, the hygrothermal properties of six insulation materials is determined; four are commercially available materials while the other two are experimental materials based on crop byproducts and natural binders. The influence of relative humidity on such properties is analysed. Moreover, the experimental Moisture Buffer Values are obtained for the six insulations, according to the protocol of the standard ISO 24353. Finally, a mass and heat coupled model is numerically solved to simulate this protocol for two of the materials, obtaining a good agreement with the experimental results.
\end{abstract}

Keywords: bio-based materials, thermal insulation, hygrothermal performance, moisture buffering, transient modelling, barley straw, corn, pith.

\section{Introduction}

The building sector is moving towards new approaches of energy efficient design, which includes not only the decrease of the thermal transmittance of the building envelope but also the improvement and use of natural and locally available building materials. In this regard, interest in bio-based insulation materials is increasing because of the generally lower environmental impact of these materials 
compared to inorganic or petroleum based insulation materials [1]. Their low embodied energy, their biodegradability and their nontoxic nature are some of their environmental benefits [2].

In terms of their hygrothermal performance, probably the most relevant property of bio-based insulation materials is their high hygroscopicity. This means that such materials have the capacity to accumulate or release moisture in their internal porous structure by adsorption or desorption from the environment [3]. The amount of moisture accumulated is material specific and dependent on the relative humidity and the temperature of the environment. Thus, hygroscopic materials can be regarded as dynamic multi-phase systems (solid, the material matrix; liquid, the water adsorbed to the surface of the material; and gas, the air and water vapour within the pores of the material).

The above mentioned characteristic is relevant because all the basic thermal and hygric properties of hygroscopic materials depend on the moisture content [4]. Collet and Pretot [5] found a rate variation of thermal conductivity with moisture content about $0.0022 \mathrm{~W} / \mathrm{mK}$ for each $1 \%$ of change in $\mathrm{RH}$ for hemp lime of between 0.090 and $0.160 \mathrm{~W} / \mathrm{mK}$, with little differences due to density and fibre/binder ratio. Other authors reported the variation of thermal conductivity with temperature to be from one to two orders of magnitude lower [6,7]. Thermal diffusivity is reported to be sensitive to moisture content as well. Carmeliet et al. [8] reported a reduction of 5 to $13 \%$ when the moisture content was doubled for brick and calcium silicate respectively. Jerman et al. [9] reported a rate variation of water vapour permeability of $0.1310^{-6} \mathrm{~m}^{2} / \mathrm{s}$ for each $1 \%$ of change in $\mathrm{RH}$ for an aerated concrete with a water vapour permeability (dry cup) of $2.17 \cdot 10^{-6} \mathrm{~m}^{2} / \mathrm{s}$. Finally, heat capacity significantly increases with moisture content due to the high specific heat capacity of water [4].

A certain amount of energy is associated with the adsorption process and the phase change of adsorbed moisture which also has implications for the thermal performance of the material. Therefore, it is clear that hygroscopicity has a direct influence on the energy performance of buildings. The extent and orientation (against or in favour of building energy efficiency) of such influence depends on each specific case and how this phenomenon is taken into account during the design and construction phases.

The understanding of the combined mechanisms of heat and mass transport in porous media is essential in the quantification of the thermal building performance. Different models have been proposed to describe the dynamic evolution of hygroscopic materials under variable environmental conditions of relative humidity and/or temperature [10-13]. Although there are some differences between the various models, all of them are based on the conservation of mass and energy. In their corresponding equations, there are some magnitudes (thermal conductivity, density, specific heat, water vapour permeability) that depend on relative humidity, and therefore a complete knowledge of the material hygrothermal properties is needed in order to numerically solve the equations. 
The capacity of adsorbing and desorbing moisture enables hygroscopic materials to act as a moisture buffer, moderating extremes of humidity in an indoor environment [14-16]. This has positive effects on indoor air quality and might enable a reduction of the ventilation rate and thus, of heat loses. The moisture buffer performance of a room is the ability of the materials within the room to moderate variations in the relative humidity. The NORDTEST Project [17,18] and the Japanese Industrial Standard JIS A 1470-1 (2002) introduced a useful index to quantify the moisture buffer capacity of a material in conditions of surrounding humidity variation. The Moisture Buffer Value (MBV) indicates the amount of water vapour that is transported in or out of a material, when the sample is exposed to cyclic step-changes in relative humidity between high and low values for determined periods of time. A related index is included in the international standard ISO 24353. It is a material/environment characterisation, and thus is affected by air speed and depends on the air surface resistance $[17,19]$. The MBV is normalized per $\%$ of relative humidity variation, and its units are $\mathrm{kg} /\left(\mathrm{m}^{2} \% \mathrm{RH}\right)$.

In the present work the hygrothermal properties of six insulation materials (four commercially available and two experimental materials) are compared. The dependence of thermal conductivity, thermal diffusivity and water vapour permeability with relative humidity is experimentally determined. Experimental Moisture Buffer Values are obtained for the six insulations, and these values are compared with numerical results obtained from a dynamical model.

\section{Dynamical model}

The heat and mass transport in porous media can be modelled, under certain assumptions, by a set of one-dimensional coupled equations [14] which account for conservation of mass (adsorbed and gas phases) and energy:

$\rho_{l} \frac{\partial \varepsilon_{l}}{\partial t}+\dot{m}=0$

$\frac{\partial\left(\rho_{v} \varepsilon_{g}\right)}{\partial t}-\dot{m}=\frac{\partial}{\partial x}\left(D_{v} \frac{\partial \rho_{v}}{\partial x}\right)$

$\rho C_{p} \frac{\partial T}{\partial t}+\dot{m} h_{a d}=\frac{\partial}{\partial x}\left(\lambda \frac{\partial T}{\partial x}\right)$

where $t$ is the time and $x$ the spatial position along the width of the sample. In these equations $\varepsilon_{1}(x, t)$ and $\varepsilon_{\mathrm{g}}(\mathrm{x}, \mathrm{t})$ are the volume fractions of liquid and gas phases respectively, $\rho_{\mathrm{v}}(\mathrm{x}, \mathrm{t})$ is the vapour water density and $\mathrm{T}(\mathrm{x}, \mathrm{t})$ is the temperature. Volume fractions are constrained by the relationship $\varepsilon_{1}(\mathrm{x}, \mathrm{t})+\varepsilon_{\mathrm{g}}(\mathrm{x}, \mathrm{t})+\varepsilon_{\mathrm{s}}=1$, where $\varepsilon_{\mathrm{s}}$ is the volume fraction of the solid walls of the material. $\rho_{\mathrm{l}}$ is the liquid water density and $h_{a d}$ is the latent heat of sorption. From each pair of local and instantaneous values of 
vapour density and temperature, the relative humidity $\phi$ can be evaluated by using thermodynamic relationships. $\phi=\mathrm{Pv} / \mathrm{Psat}$, where $\mathrm{Pv}$ is the vapour pressure and Psat the saturation vapour pressure at temperature $T$. The vapour diffusion coefficient, $D_{v}$, is evaluated from the water vapour permeability $\delta$ as $\mathrm{D}_{\mathrm{v}}=\delta \mathrm{Rv} \mathrm{T}$, with $\mathrm{Rv}$ being the gas constant for water vapour. The magnitudes $\lambda$ (thermal conductivity), $\delta$ (water vapour permeability) and $\rho \mathrm{C}_{\mathrm{p}}$ (product of density by heat capacity of the sample) depend on the relative humidity, so they need to be re-evaluated each time step. $\dot{m}(x, t)$ is the phase change rate per unit volume and can be evaluated from the moisture content, $\mathrm{u}$, as:

$\dot{m}=-\rho_{0} \frac{\partial u}{\partial t}$

where $\rho_{0}$ is the dry density of the material. Moisture content $u$ depends on relative humidity according to its corresponding sorption isotherm curve. In conclusion, in order to use Eqs. (1)-(4), there are four magnitudes which dependence with relative humidity should be known: $u, \lambda, D_{v}$, and $\rho C_{p}$. In the next section, these dependences have been experimentally obtained for six insulation materials.

\section{Materials and methods}

\subsection{Materials and samples}

Samples of six different bio-based insulation materials were used. Four of them, namely, hemp lime (HL), hemp fibre (HF), wood wool (WW) and wood fibre (WF), are commercially available thermal insulations, while the barley straw-starch (BS) and corn pith-alginate (CA) are experimental insulation materials. Formulations for the latter were optimized in previous work in order to obtain suitable composites [20]. The basic properties and composition of the tested materials are listed in Table 1.

Table 1. Basic information about the materials used in the investigation.

\begin{tabular}{cclrc}
\hline Sample & $\begin{array}{c}\text { Board } \\
\text { type }\end{array}$ & \multicolumn{1}{c}{ Composition } & $\begin{array}{c}\text { Dry } \\
\text { density } \\
\left(\mathbf{k g} / \mathbf{m}^{3}\right)\end{array}$ & $\begin{array}{c}\text { Porosity } \\
(\mathbf{a . u .})\end{array}$ \\
\hline CA & Rigid & Corn pith (89\%), sodium alginate (11\%) & 48.1 & 0.98 \\
BS & Rigid & Barley straw (81\%), corn starch (19\%) & 107.5 & 0.92 \\
HF & Flexible & Hemp fibre, adhesive, additives & 41.1 & 0.97 \\
HL & Rigid & Hemp hurds (50\%), lime (50\%) & 286.0 & 0.83 \\
WW & Flexible & Wood wool, adhesive, additives & 60.2 & 0.96 \\
WF & Rigid & Wood fibre, additives & 212.2 & 0.86 \\
\hline
\end{tabular}




\subsection{Thermal conductivity and diffusivity}

With the aim to determine the influence of relative humidity on thermal conductivity and thermal diffusivity of the insulation materials, samples of $200 \times 200 \times 40 \mathrm{~mm}$ were prepared. They were conditioned at different relative humidities at $20^{\circ} \mathrm{C}$ in sealed capsules containing saturated salt solutions for at least two weeks. Their thermal conductivity and thermal diffusivity was then determined with the Quickline-30 Electronic Thermal Properties Analyser using a surface probe with a disk sensor. Such equipment is based on the analysis of the temperature response of the material to heat flow impulses induced by electrical heating using a resistor heater having direct thermal contact with the surface of the sample. The accuracy of measurement was $5 \%$ of reading $+0.003 \mathrm{~W} / \mathrm{mK}$. In order to maintain the environmental conditions during the test, the surface probe was introduced into the capsules for testing. After each measurement the moisture content of the samples was gravimetrically determined with an accuracy of $0.01 \mathrm{~g}$. At the end of the test the samples were oven dried at $100^{\circ} \mathrm{C}$ for $24 \mathrm{hs}$ and weighed again in order to quantify the dry mass. From thermal conductivity $(\lambda)$ and thermal diffusivity $(\alpha)$, the value of $\rho C_{p}$ was determined by the relation (Eq 5):

$$
\rho C_{p}=\frac{\lambda}{\alpha}
$$

The results obtained with the Quickline-30 were compared with those determined using a Heat Flow Meter Laser Comp FOX 600. operating on the principle of the stationary slab method (BS EN 12664). Uncertainty in the thermal conductivity measurement may be influenced by different sources, but can be estimated within $\pm 1.5 \%$. The mean temperature was, $20{ }^{\circ} \mathrm{C}$ with a $20^{\circ} \mathrm{C}$ temperature gradient. Measurements were done both on dry specimens and on specimens conditioned at $80 \%$ relative humidity. In order to maintain and control the moisture content of the specimens during the test, they were wrapped with a plastic film and weighed before and after the test. The steady-state equilibrium criteria were that the heat flux should not vary more than $2 \%$ of 10 successive blocks, with a minimum 100 blocks in total, each block being approximately 6 minutes, while the temperature of each plate remained within $0.2^{\circ} \mathrm{C}$ of the setpoint..

\subsection{Water vapour permeability $(\delta)$}

The water vapour permeability of the materials $(\delta)$ was determined experimentally using the cup method, by creating a relative humidity gradient across the samples (ISO 12571). In order to determine the influence of relative humidity on water vapour permeability, at least two tests were performed, one at a low mean relative humidity (dry cup) and the other at higher relative humidity (wet cup). To this end, $100 \times 100 \mathrm{~mm}$ samples, conditioned at room conditions $\left(20^{\circ} \mathrm{C}\right.$ and $60 \mathrm{RH}$ ), 
were placed on top of sealed cases containing a silica gel (dry cup, RH $9.0 \pm 0.1 \%$ ) or a saturated water solution of ammonium chloride (wet cup, $\mathrm{NH}_{4} \mathrm{Cl}, \mathrm{RH} 79.3 \% \pm 0.1 \%$ ). This salt was chosen because the highly absorptive properties of the materials meant that they would take many months to reach hygric equilibrium at a higher relative humidity. The thickness of the air layer between the sample and salt solution was $15 \mathrm{~mm}$ at the beginning of the test but increased not beyond $20 \mathrm{~mm}$ as water evaporated. The resistance of the air layer was not taken into account for calculation, assuming that the true permeability of the materials may be slightly lower due to the effect of the air layer. The sides of the samples were sealed so that only their top and their bottom surfaces were exposed. The arrangements were placed in a conditioning chamber at $20 \pm 1{ }^{\circ} \mathrm{C}$ and $60 \pm 3 \%$ relative humidity and protected with a meshed cover in order to prevent air movement. The difference in partial vapour pressure at each side of the samples creates a vapour flux. Specimens and cases were weighed regularly until a steady-state vapour flux was established. A linear regression was used to determine the water vapour flow rate, $\mathrm{G}(\mathrm{kg} / \mathrm{s})$. The water vapour permeability was then calculated using the following expression:

$$
\delta=\frac{G d}{A \cdot \Delta p}
$$

where, $\mathrm{d}(\mathrm{m})$, is the sample thickness, $\mathrm{A}\left(\mathrm{m}^{2}\right)$, is the exposed surface area, and $\Delta \mathrm{p}(\mathrm{Pa})$ is the average water vapour pressure difference across the sample during the test. The $\mu$ value (which expresses the ratio between the water vapour permeability of the material and that of the air) was also calculated.

\subsection{Moisture Buffering Test}

The Moisture Buffering Value has been determined using a set up designed following the methodology indicated in ISO 24353. The specimens were exposed to a series of step changes in relative humidity between two levels, in cycles of 24 hours (12hours at a 53\% RH and 12 hours at $75 \% \mathrm{RH})$ and maintaining the temperature at $23^{\circ} \mathrm{C}$. The air speed in the surrounding of the specimens during the test was measured and was typically $0.37 \mathrm{~m} / \mathrm{s}$. Specimens were prepared measuring $200 \mathrm{x}$ $200 \mathrm{~mm}$ and of a thickness representative of the product. The lateral and bottom surfaces of the samples were sealed with an aluminium foil so that only the top surface was exposed before conditioning them at $20^{\circ} \mathrm{C}$ and $60 \% \mathrm{RH}$. The samples were placed on top of a scale in order to register mass change during the test which continued until the average of the sorbed and desorbed mas $(\Delta m)$ did not change by more than 5\% over 3 cycles [17] and thus a dynamic equilibrium was reached. Moreover, the temperature at $15 \mathrm{~mm}$ below the exposed surface was also monitored during the test. MBV was calculated using the following equation:

$$
M B V=\frac{\Delta m}{A \cdot \Delta R H}
$$


where, $\Delta m$ is an average of the adsorbed and desorbed mass after the system has reached a dynamic equilibrium $(\mathrm{kg}), A$ is the exposed surface area $\left(\mathrm{m}^{2}\right)$, and $\Delta \mathrm{RH}$ is the difference in relative humidity $(\%)$.

\subsection{Numerical simulations}

The coupled partial differential Equations (1)-(4) were discretized using a finite difference approach. A one-dimensional system of size $\mathrm{L}$, with spatial step $\Delta \mathrm{x}=0.001 \mathrm{~m}$, was considered. In order to reproduce the experimental protocol described in section 3.4, the system is initialized with the values corresponding to a temperature of $23^{\circ} \mathrm{C}$ and a relative humidity of $60 \%$. Then, environmental $\mathrm{RH}$ is changed cyclically between $53 \%$ and $75 \%$ every 12 hours. The boundary conditions are convection heat and moisture transfer above one of the sample surface and an impermeable and adiabatic boundary condition below the other. The humidity dependence of the hygrothermal parameters $\lambda, \rho c_{p}$ and $\delta$, previously determined in sections 4.1 and 4.2, are used. In addition to these parameters, the moisture content as a function of the relative humidity (isotherm curves) is also introduced in the model (Eq. (4)). Such experimental data, were taken from literature and previous work [20,21], and fitted using the Oswin model, which has been reported to provide satisfactory fitting in several works [22-25]:

$$
u=A\left[\frac{\phi}{1-\phi}\right]^{B}
$$

where the fitting parameters A and B are specific to each material.

\section{Results and discussion}

\subsection{Hygrothermal properties.}

\subsubsection{Influence of relative humidity and temperature on thermal conductivity}


The thermal conductivity of the six different materials is analysed on both dry and conditioned samples with a surface probe. Results indicate a clear dependence of the thermal conductivity with density, regardless the kind of raw material tested, which is in accordance with the literature [26]. CA and WW were the materials with the lowest thermal conductivity and HL and WF the ones with the highest. For the commercially available materials the results are in agreement with declared values. The variation of $\lambda$ and $\rho C_{p}$ with relative humidity (expressed in $\%$ ) is shown in Figures 1-2. A linear regression was used to fit the experimental data $\left(\lambda=a_{\lambda}+b_{\lambda} \cdot \phi\right.$ and $\rho C p=a_{\rho C p}+b_{\rho C p} \cdot \phi$ respectively) where $\phi$ is the relative humidity expressed as the decimal ratio Pv/Psat. The values of the fitted factors are presented in Table 2.

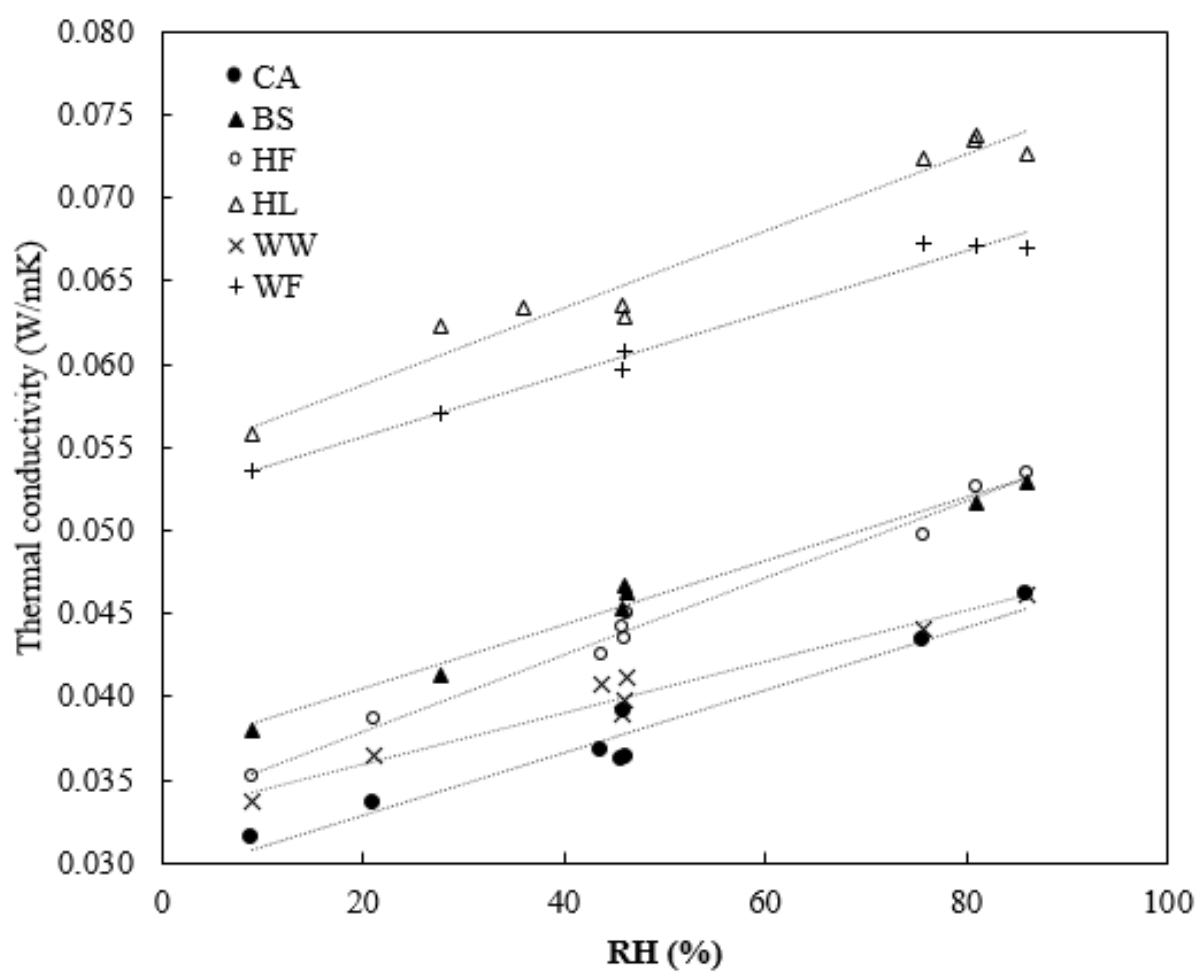

Figure 1. Variation of thermal conductivity with relative humidity for the six samples. Experimental results are linearly fitted. 


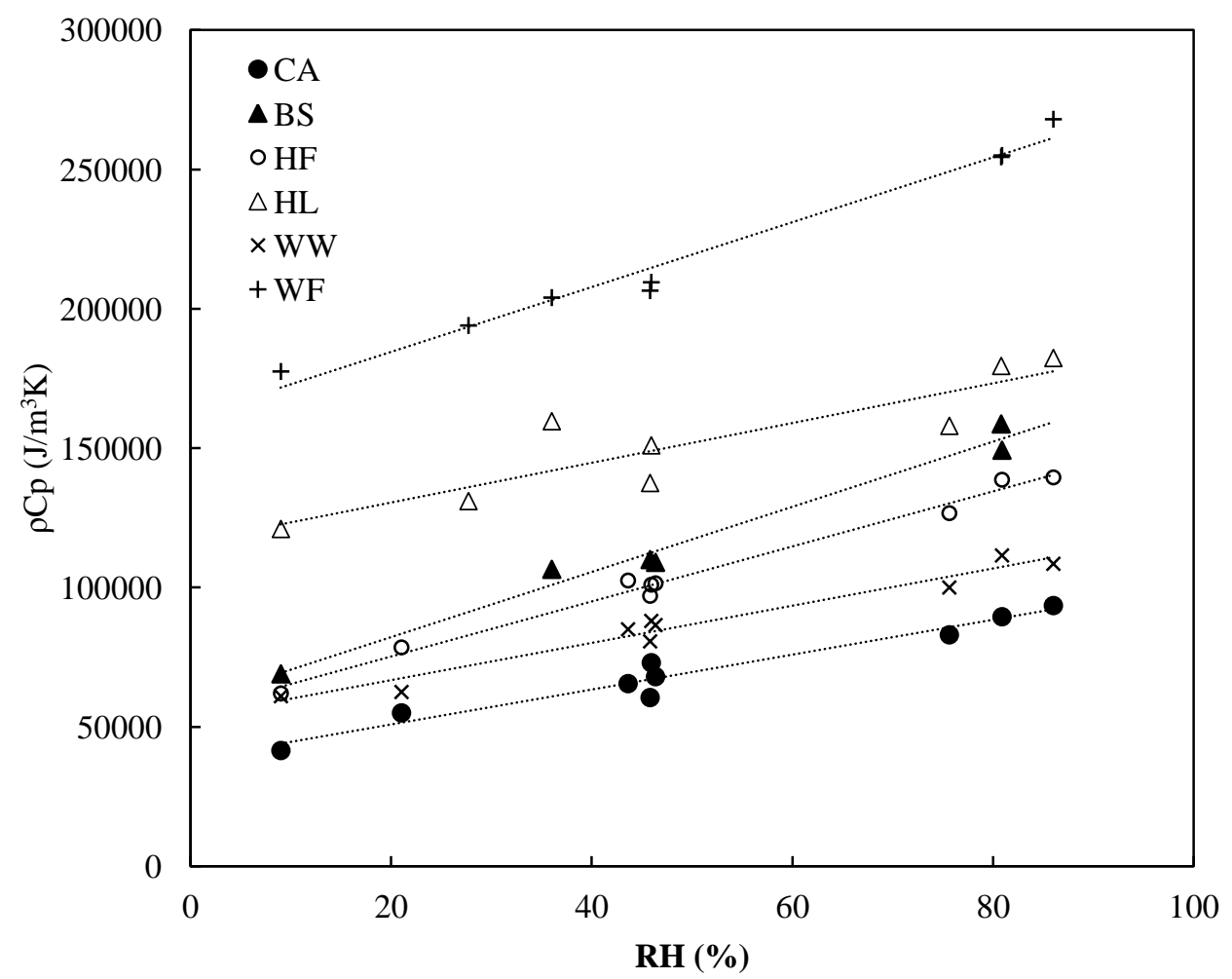

Figure 2. Variation of the product $\rho$ Cp with relative humidity for the six samples. Experimental results are linearly fitted.

Table 2. Values of the fitted factors for the $\lambda$ and. $\rho C p$ variation. with relative humidity.

\begin{tabular}{cccrr}
\hline & $\mathrm{a}_{\lambda}(\mathrm{W} / \mathrm{mK})$ & $\mathrm{b}_{\lambda}(\mathrm{W} / \mathrm{mK})$ & $\mathrm{a}_{\rho c_{\mathrm{p}}}\left(\mathrm{J} / \mathrm{m}^{3} \mathrm{~K}\right)$ & $\mathrm{b}_{\rho c_{p}}\left(\mathrm{~J} / \mathrm{m}^{3} \mathrm{~K}\right)$ \\
\hline $\mathrm{CA}$ & 0.029 & 0.019 & 38400 & 62500 \\
$\mathrm{BS}$ & 0.037 & 0.019 & 58900 & 116900 \\
$\mathrm{HF}$ & 0.033 & 0.023 & 55400 & 98800 \\
$\mathrm{HL}$ & 0.054 & 0.023 & 116200 & 71400 \\
WW & 0.033 & 0.015 & 53400 & 66700 \\
WF & 0.052 & 0.019 & 161200 & 116400 \\
\hline
\end{tabular}

As expected, in all cases the thermal conductivity increases with relative humidity. Results indicate that the changing rate of thermal conductivity in hemp composites is higher than in the rest of the materials, which implies a higher sensitivity of the materials to moisture changes. This can be observed from the slopes of the fitting equations. On the contrary, the thermal conductivity of CA and WW composites is less affected by moisture changes. The changing rate of hemp composites is $50 \%$ higher than that of corn or wood wool. Moreover, the variation of $\rho \mathrm{Cp}$ with relative humidity is also lower for CA and WW composites.

Important variations in measurements made under the same conditions on consecutive days were observed (see Figures 1-2). This might be due to moisture migration through the material when the 
sealed capsules were opened to introduce the probe and weigh the samples. Another factor that might explain such variability is the measurement method, which only analyses a part of the material near the surface of the probe through electric impulses that might also cause moisture migration.

The results of the thermal conductivity tests obtained with the electronic thermal analyser were compared to the values obtained with the Heat Flow Meter following the methodology described in the standard BS EN 12664. The samples were wrapped with a plastic film before the test in order to prevent moisture migration. Samples were weighed before and after being tested at the HFM showing a variation in mass between $0.02 \%$ and $3.6 \%$. From the isotherms presented further on, in Section 5.2 , it is evident that at low $\mathrm{RH}$, such variation corresponds to a range of up to $37 \% \mathrm{RH}$, thus causing in an important error. This indicates that the electronic thermal analyser yielded more favourable results as samples could be tested directly in the conditioning chamber. Moisture migration, within the sample, due to the established temperature gradient required during Heat Flow Meter thermal testing leads to errors in results. Additionally, associated convective transport of heat by the flowing fluids further complicates the accurate determination of thermal conductivity under steady-state test methods. Accordingly, transient thermal analysis methods are more suited to determination of thermal conductivity of moist material samples. However, at the relatively low mean test temperature and small $\left(20^{\circ} \mathrm{C}\right)$ temperature gradient, and for tests conducted extended periods, relative to dry samples, errors associated with moisture migration and convective transport have been shown to be low for RH $<70 \%$ [27] although this value is dependent on the porosity and other inner structure characteristics of the material. At low $\mathrm{RH}$, the heat flow meter returns about $11-12 \%$ higher values of thermal conductivity than the electronic thermal analyser. However, this tendency is inverted in samples conditioned at $80 \%$ relative humidity (with exception of HL), where the difference between methods is up to $25 \%$.

Table 3. Thermal conductivity measured on dry and conditioned samples with the heat flow meter (HFM) compared to the electronic thermal analyser (ETA) results.

\begin{tabular}{cccc}
\hline & $\lambda(\mathrm{W} / \mathrm{mK})$ & $\lambda(\mathrm{W} / \mathrm{mK})$ & $\lambda(\mathrm{W} / \mathrm{mK})$ \\
& HFM DRY & HFM 80\% & ETA 50\% \\
\hline CA & 0.038 & 0.038 & 0.037 \\
BS & 0.042 & 0.046 & 0.046 \\
HF & 0.040 & 0.040 & 0.044 \\
HL & 0.064 & 0.075 & 0.063 \\
WW & 0.038 & 0.039 & 0.039 \\
WF & 0.054 & 0.054 & 0.060 \\
\hline
\end{tabular}

\subsubsection{Water vapour permeability $(\delta)$}

Results of the water vapour diffusion resistance tests are shown in Table 5. Since it was determined experimentally for only two mean water vapour pressures, the fitting equation is simplified to a linear 
fitting $\left(\delta=\mathrm{a}_{\delta}+\mathrm{b}_{\delta^{\prime}} \phi\right)$, assuming that such approximation is valid only to relative humidities up to $70 \%$. The fitting parameters are shown as well in Table 4. Results show that the material with highest water vapour permeability is WW $(\mu=2.4)$ and the one with lowest is WF $(\mu=6.5)$. Thus, the two wood based materials have a different behaviour, although the rate at which permeability increases with RH is similar. Conversely, the two hemp-based materials present a similar $\mu$ value, but a distinct changing rate. The water vapour permeability of the CA specimens is highly dependent on RH: it increases almost 4 times faster than in the case of the BS samples and 1.2 times faster than the HF materials. The differences between the specimens are reduced at high RH.

Table 4. Water vapour permeability for each sample, measured by the method of the dry and wet cup.

\begin{tabular}{ccccccc}
\hline \multirow{2}{*}{ Abrev } & \multicolumn{2}{c}{ DRY $(35 \%$ mean RH $)$} & \multicolumn{2}{c}{ WET $(70 \%$ mean RH $)$} & \multicolumn{2}{c}{ Fitting factors $\left(10^{-11} \mathrm{Kg} / \mathrm{msPa}\right)$} \\
\cline { 2 - 7 } & $\delta\left(10^{-11} \mathrm{Kg} / \mathrm{msPa}\right)$ & $\mu$ & $\delta\left(10^{-11} \mathrm{Kg} / \mathrm{msPa}\right)$ & $\mu$ & $\mathrm{a}_{\delta}$ & $\mathrm{b}_{\delta}$ \\
\hline CA & $3.4 \pm 0.5$ & 5.7 & $6.0 \pm 0.6$ & 3.2 & 0.8 & 7.4 \\
BS & $5.1 \pm 0.5$ & 3.9 & $5.8 \pm 0.4$ & 3.4 & 4.4 & 2.0 \\
HF & $6.7 \pm 0.5$ & 3.0 & $8.7 \pm 0.5$ & 2.3 & 4.7 & 5.7 \\
HL & $6.6 \pm 0.5$ & 3.0 & $7.9 \pm 0.5$ & 2.5 & 5.3 & 3.7 \\
WW & $8.1 \pm 0.5$ & 2.4 & $9.5 \pm 0.4$ & 2.1 & 6.7 & 4.0 \\
WF & $3.0 \pm 0.3$ & 6.5 & $4.7 \pm 0.5$ & 4.2 & 1.3 & 4.9 \\
\hline
\end{tabular}

\subsection{Moisture Buffering}

\section{2..1 Experimental results}

The mass change of the six samples over time at dynamic equilibrium is shown in Figure 3. Moreover, Table 5 shows the results of moisture uptake once the dynamic equilibrium was reached and the moisture buffering values (MBV) of the different specimens. The values shown correspond to the mean values of three cycles. It is important to note that although the error due to mass change at three consecutive cycles at dynamic equilibrium is typically $3 \%$, a higher error is introduced due to uncontrolled variations in the exposed area. Due to the nature of the tested materials, which are in the form of flexible mats, present surface irregularities and swell during the test due to water sorption, a tolerance of $1 \mathrm{~mm}$ per side has to be assumed. This results in an error of between 12\% and 15\% for the MBV presented. The results show significant differences in the MBV of the materials. HL and BS were found to be the materials with a higher moisture buffering capacity $\left(3.3 \mathrm{~g} / \mathrm{m}^{2} \cdot \Delta \mathrm{RH}\right.$ and 3.2 $\mathrm{g} / \mathrm{m}^{2} \cdot \Delta \mathrm{RH}$ respectively). For HL, this value is a bit higher than that reported in literature (between 2.0 and $\left.2.5 \mathrm{~g} / \mathrm{m}^{2} \cdot \Delta \mathrm{RH}\right)$, although the use of different climate chamber conditions make it difficult to compare results that use different protocols [28]. Also, small differences in air movement across the sample surface can have a significant effect on the result. Other studies of HL have also used different formulations and manufacturing techniques. The hygrothermal performance varies greatly depending 
on constituent ratio and level of compaction, which result in differences in density and porous structure [29].

Two other materials, CA and WW, presented a high MBV too, while the other two, HF and WF, had the lowest values, close to $2.0 \mathrm{~g} / \mathrm{m}^{2} \Delta \mathrm{RH}$. All the values correspond to materials considered to be good (between 1.0 and $2.0 \mathrm{~g} / \mathrm{m}^{2} \Delta \mathrm{RH}$ ) and excellent (from $2.0 \mathrm{~g} / \mathrm{m}^{2} \Delta \mathrm{RH}$ ) moisture buffers [30].

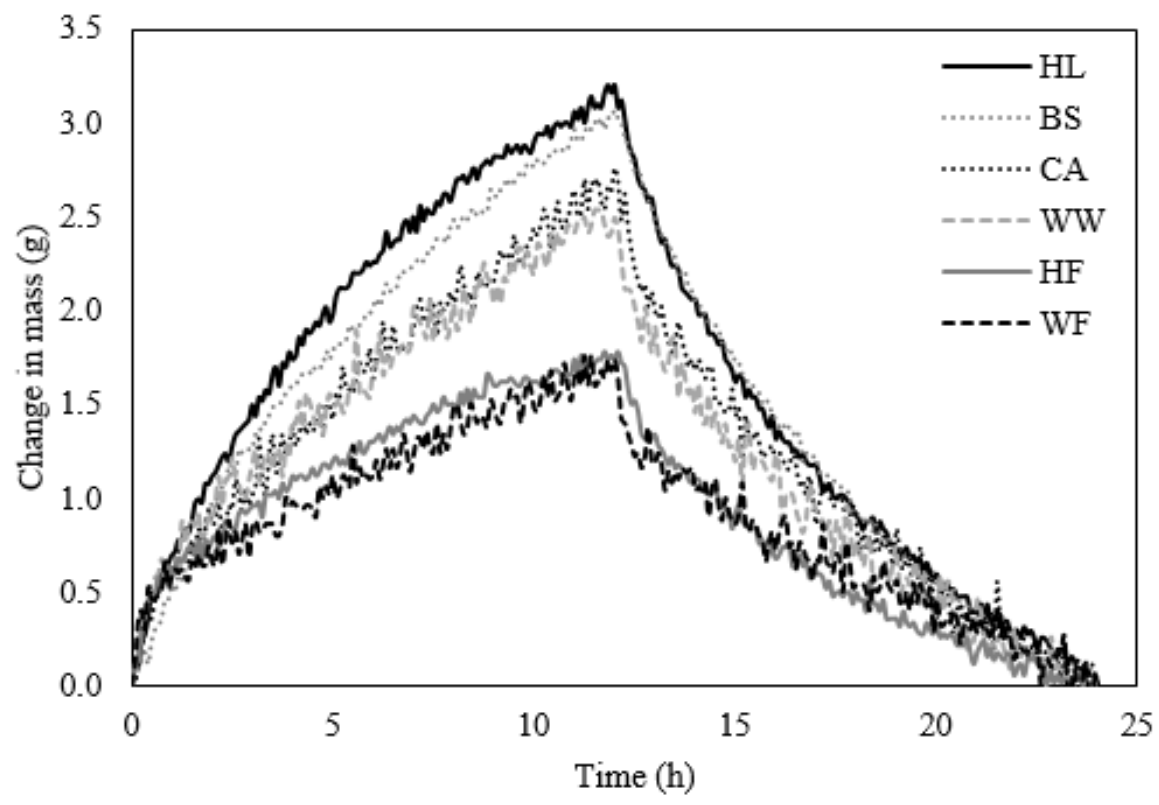

Figure 3. Change in mass of the six insulation materials over one cycle once the dynamic equilibrium is reached.

Table 5. Moisture buffering values of the insulation of the samples.

\begin{tabular}{cccc}
\hline Abrev & $\begin{array}{c}\text { Width } \\
(\mathrm{mm})\end{array}$ & $\begin{array}{c}\text { Moisture } \\
\text { uptake }(\mathrm{g})\end{array}$ & $\begin{array}{c}\text { MBV } \\
\left(\mathrm{g} / \mathrm{m}^{2} \cdot \Delta \mathrm{RH}\right)\end{array}$ \\
\hline CA & 75 & $2.91 \pm 0.09$ & 3.0 \\
BS & 96 & $3.20 \pm 0.10$ & 3.2 \\
HF & 75 & $1.87 \pm 0.05$ & 2.3 \\
HL & 90 & $3.15 \pm 0.05$ & 3.3 \\
WW & 125 & $2.50 \pm 0.10$ & 2.6 \\
WF & 80 & $1.81 \pm 0.05$ & 1.9 \\
\hline
\end{tabular}

\subsubsection{Numerical simulations}

As an example of an application of the model proposed in Section 2 a numerical simulation has been performed, according to what is explained in section 3.5, for two of the materials: a commercial one (HF) and an experimental one (CA). Materials and hygrothermal characteristics are used from tables 1, 2 and 5. The moisture content as a function of RH has been obtained from experimental data measured with a Dynamic Vapour Sorption (DVS) equipment $[20,21]$ for CA \& HF. Figure 4 shows the adsorption isotherms for the two materials. Solid lines correspond to the Oswin model curves (Eq. 
(10)), with parameters A and B obtained from fits in the range of RH between $30 \%$ and $80 \%$. These parameters A and B have been used in the numerical simulations.

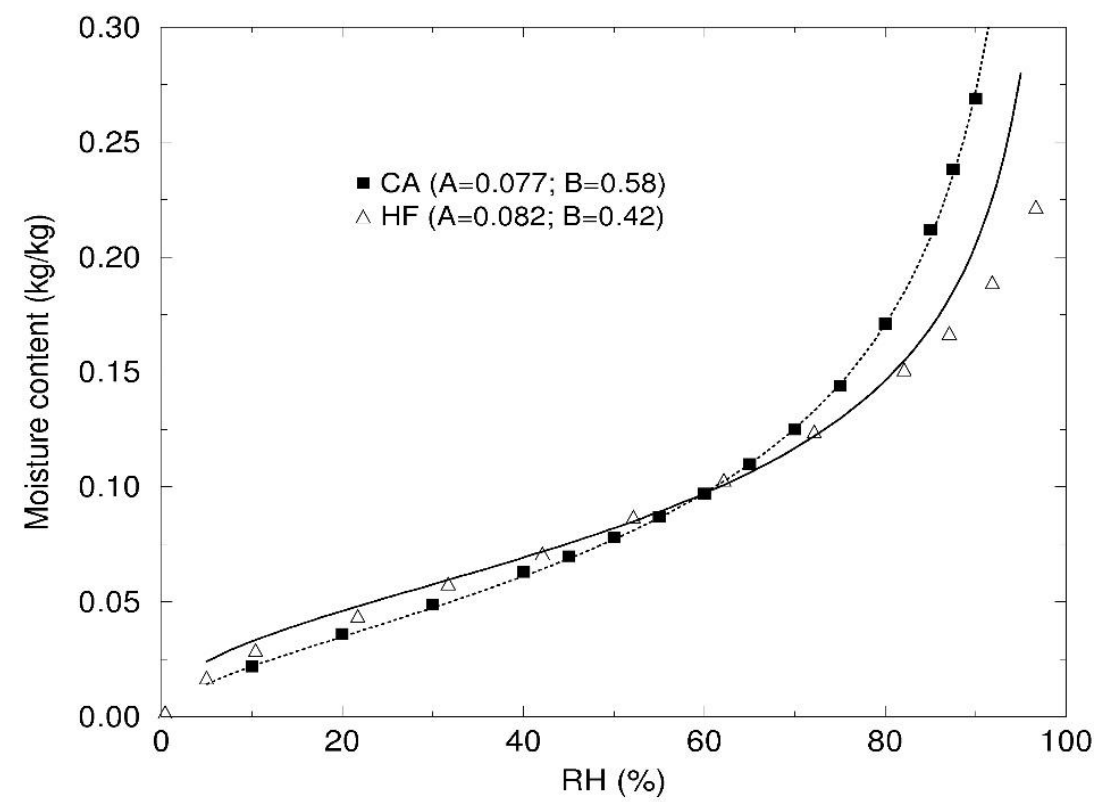

Figure 4. Moisture content of the materials at different water vapour pressures. Experimental results are fitted using the Oswin equation. The inset shows the fitting parameters $A$ and $B$

The evolution of the water vapour density, $\rho_{v}$, at different positions of the system, has been recorded during the simulations over several cycles. Figure 5 shows the results, for three cycles, at distances from the exposed surface of $15 \mathrm{~mm}, 30 \mathrm{~mm}$ and $60 \mathrm{~mm}$, for data corresponding to CA material (upper panel) and HF (lower panel). It is observed that vapour density oscillations have higher amplitude and occur at lower times for the HF material than for the CA one. This is because of the HF permeability value, which is about 2 times higher than that for CA. However, the evolution of the temperature at the same three positions, plotted in Figure 6, shows lower differences for the two materials. It is probably due to two combined and opposite effects: HF has a higher vapour permeability but a lower thermal diffusivity than CA. According to the model, temperature increases (decreases) locally when water adsorption (desorption) takes place (second term of Eq. (3)). Then, heat diffuses (third term of Eq. (3)), leading to the observed peaks. Peaks are obviously smaller in deeper positions, where vapour density oscillations have lower magnitude.

Finally, the numerical simulation results have been compared with the corresponding experiments. The temperature evolutions at a distance of $15 \mathrm{~mm}$ from the exposed surface are compared with the experimental measurements in Figure 7, obtaining a good agreement. Both experiments and simulations show a similar value for temperature peaks for CA and HF. In the case of CA, the slower changes in water vapour density observed in Figure 5 are reflected in a slower evolution of temperature The change in mass of a sample of area $0.2 \times 0.2 \mathrm{~m}^{2}$ is obtained from the simulations and compared with the experiments in Figure 8. In this case, the agreement is not so good, which is 
attributed to the error caused by inaccuracy in determining the area of the exposed surface, as discussed in Section 5.1.

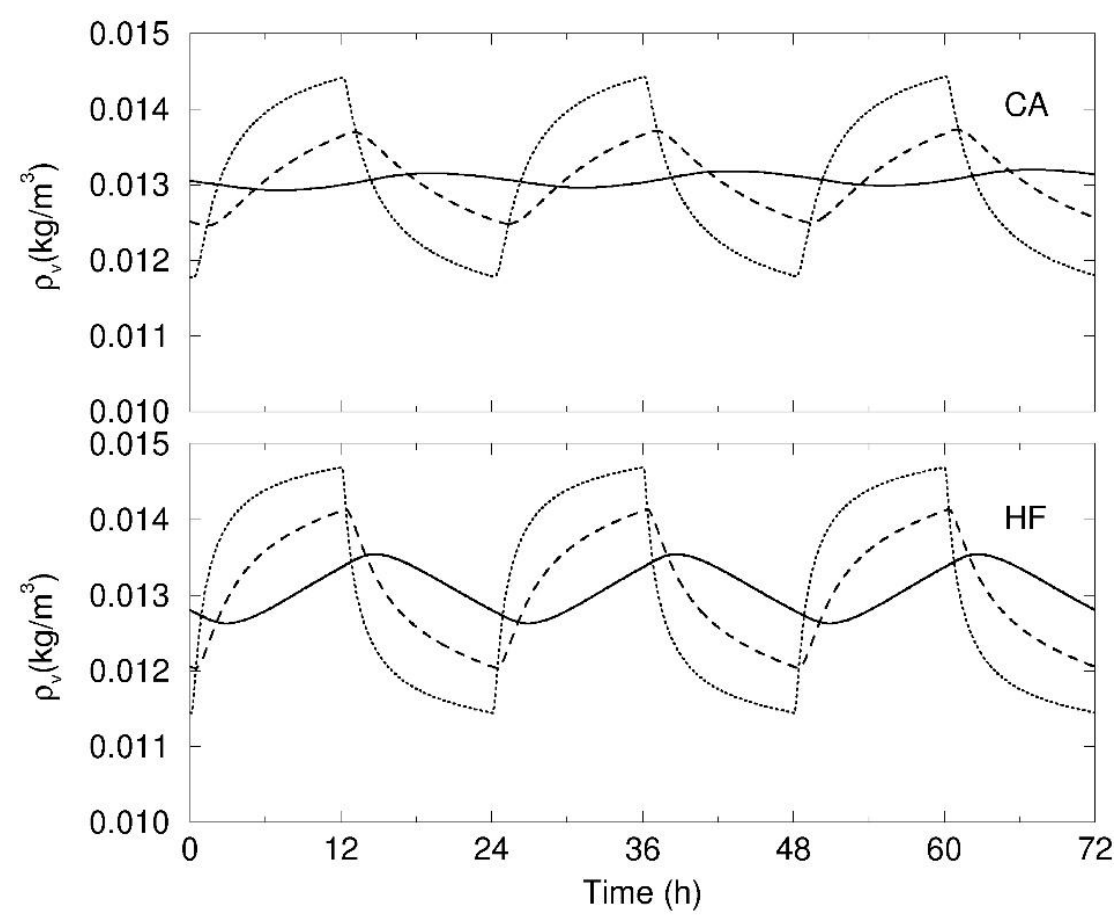

Figure 5. Water vapour density at positions $15 \mathrm{~mm}$ (dotted line), $30 \mathrm{~mm}$ (dashed line) and $60 \mathrm{~mm}$ (solid line) for CA and HF insulation materials over three cycles.

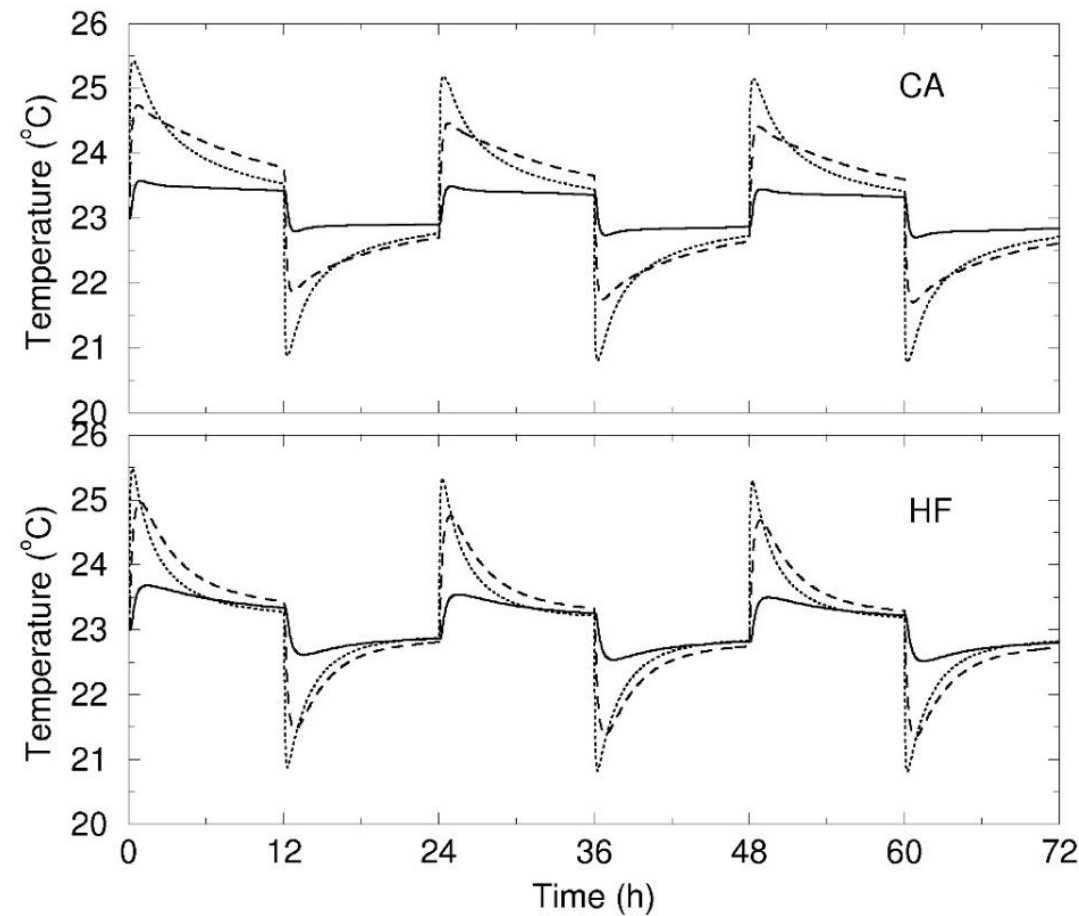

Figure 6. Temperature at positions $15 \mathrm{~mm}$ (dotted line), $30 \mathrm{~mm}$ (dashed line) and $60 \mathrm{~mm}$ (solid line) for CA and HF insulation materials over three cycles. 


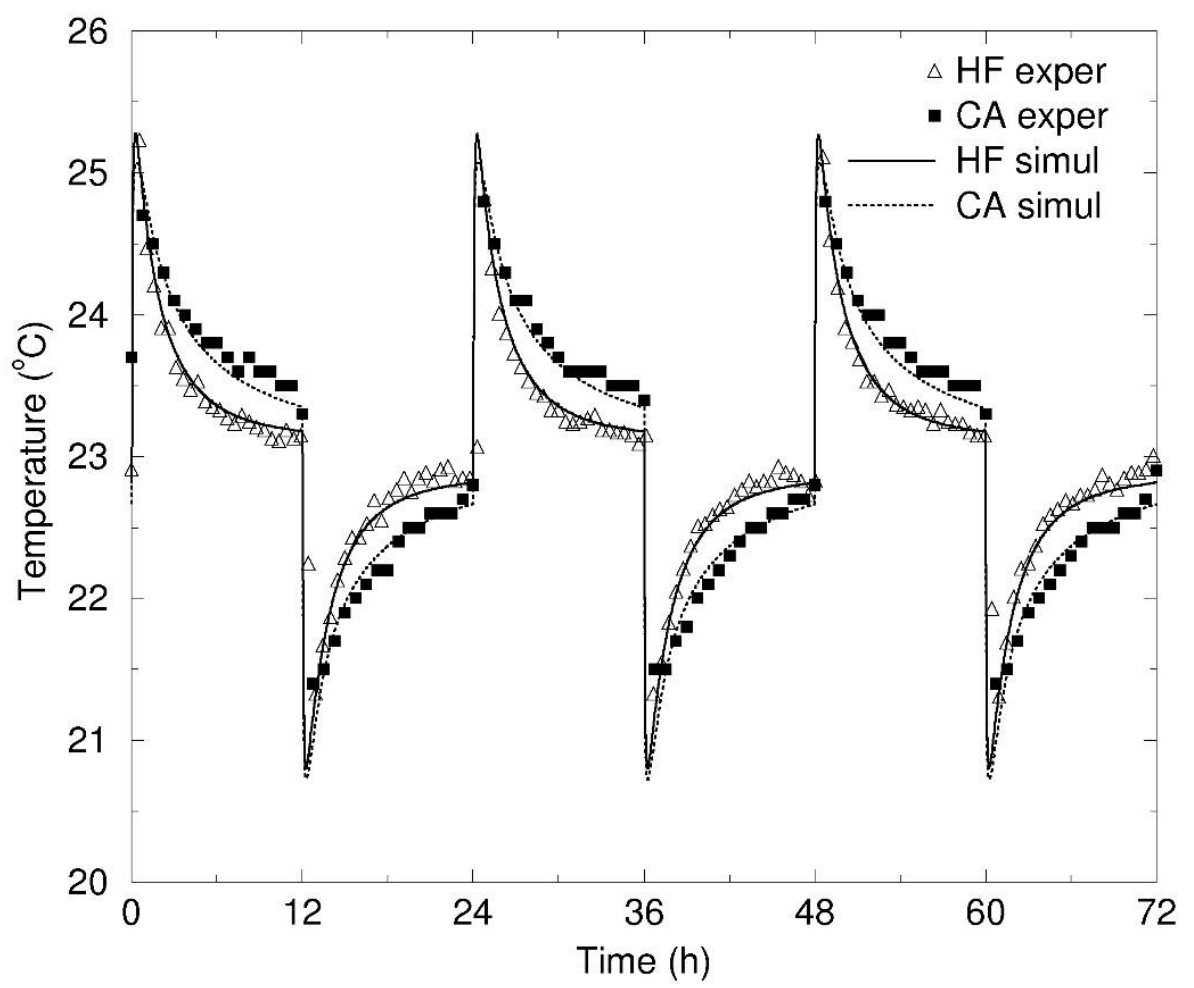

Figure 7. Temperature at position $15 \mathrm{~mm}$ for CA and HF insulation materials over three cycles once the dynamic equilibrium is reached. The experimental results (symbols) are compared to numerical simulations (lines).

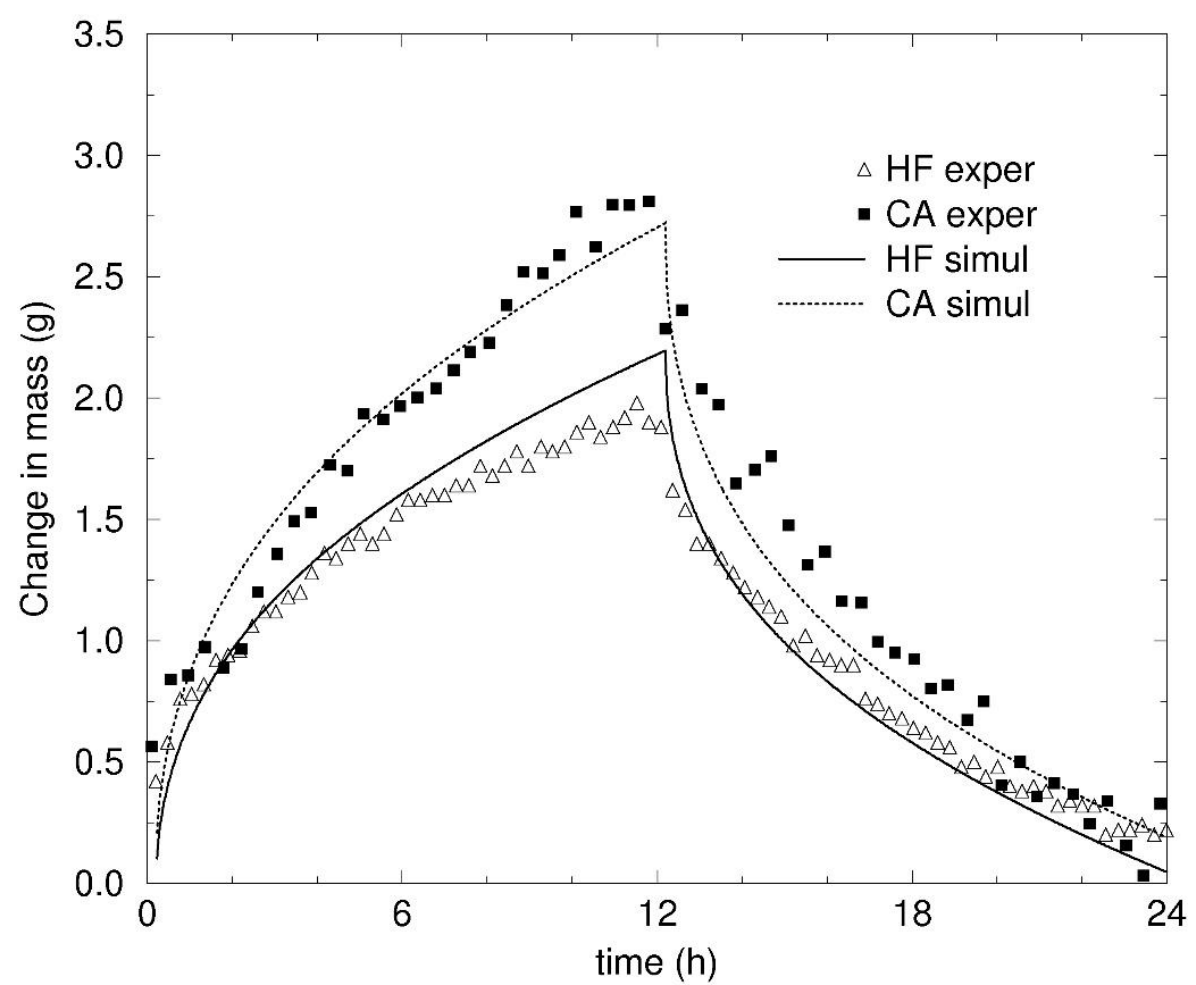

Figure 8. Change of mass of the CA and the HF insulation materials over one cycle once the dynamic equilibrium is reached. The experimental results (symbols) are compared to numerical simulations (lines). 


\section{Conclusions}

The dependence of thermal conductivity, thermal diffusivity and water vapour permeability of the six different materials with relative humidity has been experimentally determined. It has been found that the rate variation of thermal conductivity was similar for all the materials, slightly higher on hemp composites, which would indicate a higher sensitivity of such materials to changes in humidity. The analysis of the dependence of thermal conductivity on relative humidity supports the usual practice of neglecting the effect of temperature in models and calculations. However, in specific situations, such as extreme climates or façades directly exposed to solar radiation, the temperature changes might have a more important role in the hygrothermal performance of the materials. Regarding the water vapour permeability, it was found that the differences between the tested materials are shortened when the relative humidity increases. The rate variation of permeability with relative humidity was four times higher for the corn pith samples than for the barley straw insulations. HF also showed a high sensitivity to changes in RH.

The MBV was also determined. The results show three distinct behaviours. HL and BS were the materials showing the highest moisture buffering capacity. CA and WW also had a good buffering capacity. HF and WF exhibited the lowest moisture buffering capacity. These results were compared to numerical simulations. The dependence of the hygrothermal parameters on the relative humidity, found experimentally, are used in the numerical resolution of a model based on coupled equations for heat and mass transfer. Results show a quite good agreement with the experiments.

The six materials presented different hygrothermal behaviours, which indicate that the choice of the kind of bio-based material may have implications on the overall building performance. Under what conditions and to what extent this occurs, needs to be addressed in future work. To this end, not only the materials but the whole building systems need to be analysed. Indeed, the application of these materials in real buildings is an important factor to take into account when assessing their hygrothermal performance. Insulation materials are rarely in direct contact with indoor air, which significantly limits their ability to influence the indoor environment. The development of hygroscopic thermal insulation materials might explore the possibility of their use as a finishing material (as it is possible, for instance, with OSB boards) or combined with highly hygroscopic renders (such as clay or lime) which contribute to enhance the moisture buffering capacity of these materials. Numerical simulations facilitate the analysis of the effect of several factors in the different magnitudes (water vapour density, temperature) and can be a useful tool to study the implications on building performance more easily than in a real experiment. 


\section{ACKNOWLEDGEMENTS}

M.P and A.M.L would like to thank MINECO (Spain) for the support provided under the project BIA2014-52688-R. This work is also supported by Generalitat de Catalunya under the project. 2014LLAV00031 and under the PhD studentships FI-DGR. N.H, A.S. and P.W. would like to thank the EPSRC for funding this work (grant EP/J019917/1) and the following industrial partners for their support: Lime Technology, White Design Associates, Integrated Environmental Solutions, Arup, and Plant Fibre Technology. We also acknowledge the help and support of staff and students at BRE CICM, Department of Architecture and Civil Engineering at the University of Bath.

\section{REFERENCES}

[1] A. Pfundstein, M; Gellert, R; Spitzner, MH; Rudolphi, Insulating Materials. Principles, Materials, Applications, Detail Pra, Munich, 2007.

[2] R. Murphy, A. Norton, F. Alexander, Life Cycle Assessments of Natural Fibre Insulation Materials. Final Report., Defra Life. (2008).

[3] A. Korjenic, V. Petránek, J. Zach, J. Hroudová, Development and performance evaluation of natural thermal-insulation materials composed of renewable resources, Energy Build. 43 (2011) 2518-2523. doi:10.1016/j.enbuild.2011.06.012.

[4] M. Jerman, R. Cerny, Effect of moisture content on heat and moisture transport and storage properties of thermal insulation materials, Energy Build. 53 (2012) 39-46. doi:10.1016/j.enbuild.2012.07.002.

[5] F. Collet, S. Pretot, Thermal conductivity of hemp concretes: variation with formulation, density and water content, Constr. Build. Mater. 65 (2014) 612-619. doi:10.1016/j.conbuildmat.2014.05.039.

[6] F. Ochs, W. Heidemann, H. Müller-Steinhagen, Effective thermal conductivity of moistened insulation materials as a function of temperature, Int. J. Heat Mass Transf. 51 (2008) 539-552. doi:10.1016/j.ijheatmasstransfer.2007.05.005.

[7] a. Karamanos, S. Hadiarakou, a. M. Papadopoulos, The impact of temperature and moisture on the thermal performance of stone wool, Energy Build. 40 (2008) 1402-1411. doi:10.1016/j.enbuild.2008.01.004.

[8] J. Carmeliet, H. Hens, S. Roels, O. Adan, H. Brocken, R. Cerny, et al., Determination of the liquid water diffusivity from transient moisture transfer experiments, J. Build. Phys. 27 (2004) 277-305. doi:10.1177/1097196304042324.

[9] M. Jerman, M. Keppert, J. Výborný, R. Černý, Hygric, thermal and durability properties of autoclaved aerated concrete, Constr. Build. Mater. 41 (2013) 352-359. doi:10.1016/j.conbuildmat.2012.12.036.

[10] N. Mendes, F.C. Winkelmann, R. Lamberts, P.C. Philippi, Moisture effects on conduction loads, Energy Build. 35 (2003) 631-644. doi:10.1016/S0378-7788(02)00171-8.

[11] A.D. Tran Le, C. Maalouf, T.H. Mai, E. Wurtz, F. Collet, Transient hygrothermal behaviour of a hemp concrete building envelope, Energy Build. 42 (2010) 1797-1806.

doi:10.1016/j.enbuild.2010.05.016. 
[12] M. Qin, R. Belarbi, A. Ait-Mokhtar, F. Allard, Simulation of coupled heat and moisture transfer in air-conditioned buildings, Autom. Constr. 18 (2009) 624-631.

doi:10.1016/j.autcon.2008.12.006.

[13] O.F. Osanyintola, Transient moisture characteristics of spruce plywood, Master Thesis. University of Saskatchewan, 2005.

[14] O.F. Osanyintola, C.J. Simonson, Moisture buffering capacity of hygroscopic building materials: Experimental facilities and energy impact, Energy Build. 38 (2006) 1270-1282. doi:10.1016/j.enbuild.2006.03.026.

[15] C.J. Simonson, M. Salaonvaara, T. Ojanen, Heat and mass transfer between indoor air and a permeable and hygroscopic building envelope: Part II - Verification and numerical studies, J. Build. Phys. 28 (2004) 161-185. doi:10.1177/1097196304044397.

[16] M. Qin, G. Walton, R. Belarbi, F. Allard, Simulation of whole building coupled hygrothermalairflow transfer in different climates, Energy Convers. Manag. 52 (2011) 1470-1478. doi:10.1016/j.enconman.2010.10.010.

[17] C. Rode, R. Peuhkuri, K.K. Hansen, B. Time, K. Svennberg, J. Arfvidsson, et al., Nordest project on moisture buffer value of materials, in: AIVC Conf. Energy Perform. Regul., Brussels, 21-23 Sept., 2005.

[18] C. Rode, R. Peuhkuri, B. Time, K. Svennberg, T. Ojanen, P. Mukhopadhyaya, et al., Moisture Buffer Value of Building Materials, J. ASTM Int. 4 (2007) 100369. doi:10.1520/JAI100369.

[19] M.O. Abadie, K.C. Mendonça, Moisture performance of building materials: from material characterization to building simulation using the Moisture Buffer Value concept, Build. Environ. 44 (2009) 388-401. doi:10.1016/j.buildenv.2008.03.015.

[20] M. Palumbo, Contribution to the development of new bio-based thermal insulation materials made from vegetal pith and natural binders, Universitat Politècnica de Catalunya, 2015. http://tdx.cat/handle/10803/314580.

[21] C.A. Hill, A. Norton, G. Newman, The water vapor sorption behavior of natural fibers, J. ofAppliedPolymer Sci. 112 (2009) 1524-1537. doi:10.1002/app.29725.

[22] A.V. Gálvez, E.L. Aravena, R.L. Mondaca, Isotermas de adsorción en harina de maíz (Zea mays L.), Ciência E Tecnol. Aliment. 26 (2006) 821-827. doi:10.1590/S010120612006000400017.

[23] A. Arabhosseini, W. Huisman, J. Müller, Modeling of the equilibrium moisture content (EMC) of Miscanthus (Miscanthus $\times$ giganteus), Biomass and Bioenergy. 34 (2010) 411-416. doi:10.1016/j.biombioe.2009.12.004.

[24] N.A. Aviara, O.O. Ajibola, O.A. Aregbesola, M.A. Adedeji, Moisture sorption isotherms of sorghum malt at 40 and $50^{\circ} \mathrm{C}$, J. Stored Prod. Res. 42 (2006) 290-301. doi:10.1016/j.jspr.2005.05.001.

[25] G.S. Rosa, M. a. Moraes, L. a a Pinto, Moisture sorption properties of chitosan, LWT - Food Sci. Technol. 43 (2010) 415-420. doi:10.1016/j.lwt.2009.09.003.

[26] F. Domínguez-Muñoz, B. Anderson, J. Cejudo-Lopez, A. Carrillo-Andrés, Uncertainty in the thermal conductivity of insulation materials, in: Build. Simul. 2009. Elev. Int. IBPSA Conf., Glasgow, 2009: pp. 1008-1013.

[27] D. Gawin, J. Kosny, K. Wilkes, Thermal Conductivity of Moist Cellular ConcreteExperimental and Numerical Study, Am. Soc. Heating, Refrig. Air-Conditioning Eng. (2004). http://www.ornl.org/sci/roofs+walls/staff/papers/new_90.pdf.

[28] S. Roels, H. Janssen, A comparison of the Nordtest and Japanese test methods for the moisture buffering performance of building materials, J. Build. Phys. 30 (2006) pp.137-161. 
[29] N. Holcroft, A. Shea, Effect of compaction on moisture buffering of hemp-lime insulation, in: Int. Conf. Bio-Based Build. Mater., Clermont-Ferrand, France., 2015.

[30] F. Collet, J. Chamoin, S. Pretot, C. Lanos, Comparison of the hygric behaviour of three hemp concretes, Energy Build. 62 (2013) 294-303. doi:10.1016/j.enbuild.2013.03.010. 